(c) Kluwer Academic Publishers, Dordrecht - Printed in the Netherlands

\title{
The development of the representation of conceptual knowledge in memory and the design of instruction
}

\author{
S. DIJKSTRA \\ University of Twente, P.O. Box 217, 7500 AE Enschede, The Netherlands
}

\begin{abstract}
Knowledge comprises facts, concepts and principles. Skills are categorized as either cognitive or motor skills, which are essential for solving problems. The acquisition of knowledge and skills is guided by instructions and by presenting problems to students. Firstly, the instructions for acquiring concepts, based on principles, are discussed and a model for teaching is presented. Further, the integration of class and relational concepts, together with principles, is shown to be necessary for solving problems.
\end{abstract}

\section{Introduction}

Learning is generally defined as a relatively permanent change in behavior resulting from conditions of practice. Instruction is the arrangement of a situation in such a way that the learning process will start, take place and be terminated when a given criterion is reached. This latter definition implies that investigations of learning processes should pay attention to the role of instruction in learning.

It is quite remarkable that instructional situations have not received much attention in the development of learning theory. Instructional situations have instead been defined as paradigms. For example, in learning well-defined concepts the selection paradigm means presenting the whole object population at the same time, while the reception paradigm refers to presenting the specific instances one at a time. The paradigm used strongly influences the speed with which the identification algorithm can be built up and the criterion score reached (Bruner, Goodnow and Austin, 1956; Bourne, 1970). Although learning at school includes the acquisition of a substantial number of well-defined concepts, the development of instructional design has been fairly recent.

Gagné (1965) was one of the first educational psychologists to publish an overview of the conditions of learning and who made general formulations of appropriate instruction for learning different categories of intellectual skills. An intellectual skill is one that requires the use of symbols for solving problems. It is "knowing how" or procedural knowledge (Gagné, 1985). This approach has been elaborated by Merrill $(1983,1987)$. Recently, Reigeluth (1983) defined instructional design as a discipline concerned with understanding, improving and applying methods of instruction. As such, it is a professional activity implemented by teachers and instructional developers who make decisions concerning which 
methods of instruction are best to bring about desired changes in student knowledge and skills.

Reigeluth wams of the confusion between a theory of instructional design and a theory of learning. The former focuses on methods of instruction, while the latter focuses on the learning process. This interpretation suggests that theories of instructional design and theories of learning will be developed independently. However, because the goal of instruction is to promote learning, a major question arises concerning whether these two theories - a theory of instructional design and a theory of learning - should be and can be combined.

\section{Learning task, subject matter content and instruction}

The main objective of education is the acquisition of knowledge and skills. This objective is further specified by defining the nature of the student performance and the form of the subject matter content. For example, Merrill (1983) in his model of teaching defines several categories of learning objectives using these two dimensions: performance (remember, use and find) and content (fact, concept, principle and procedure). The resulting matrix is shown in Figure 1.

Level of performance

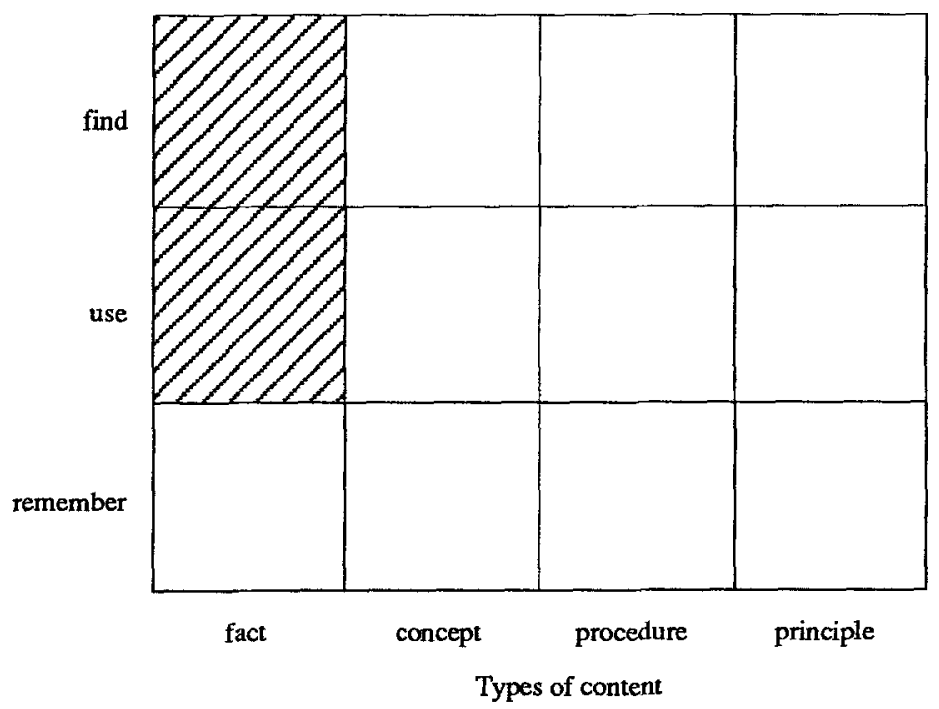

Figure 1. Performance-content matrix (from Merrill, 1983) 
Use is the performance that requires the student to apply a rule or a procedure. Find means the student should develop a rule or a procedure. Concepts are rules for categorizing objects. Principles are laws or explanations formulated in conditional or biconditional statements. For each objective indicated in a cell of the performance-content matrix, the conditions for learning a particular outcome, the nature of the behavior which has to be shown, and the criterion to be met are specified.

The matrix forms the basis for designing instruction to achieve specific learning objectives. Merrill further distinguishes between primary and secondary presentation forms. Primary presentation forms consist of two dimensions. The first dimension refers to the specificity of subject matter. Here there are two levels: a) general statements of definitions for concepts, principles and procedures, which are called generalities and $b$ ) specific cases, such as exemplars of a category or situations in which a principle is valid or a procedure can be applied, which are called instances.

The second dimension is concerned with the manner of presenting the subject matter information. This dimension also has two values: telling (expository) or questioning (inquisitory). Placing both of these dimensions, with two values each, into a matrix yields four display types.

Tell or expository Question or inquisitory

(E)

(I)

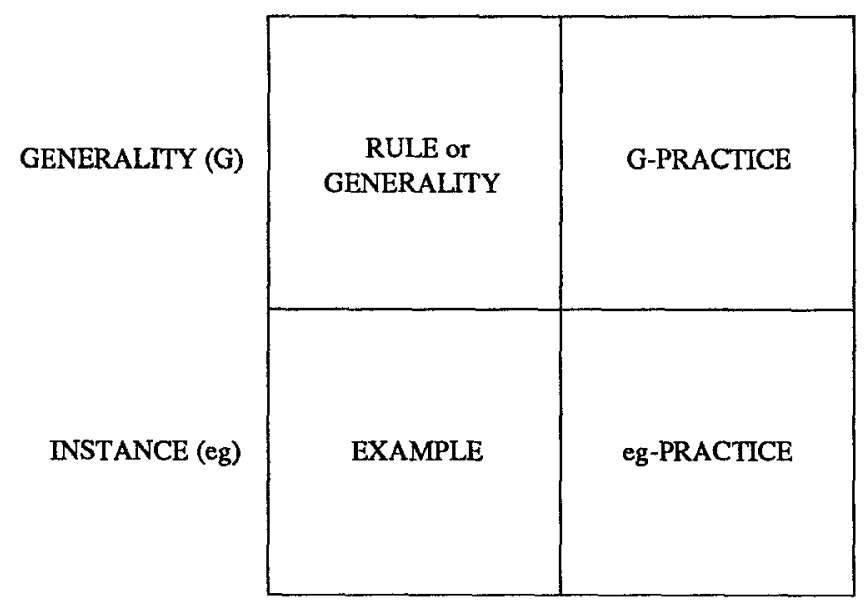

Figure 2. Primary presentation forms (from Merrill, 1983) 
The primary presentation forms are extended by the secondary presentation forms which, for the most part, are extensions of the primary presentation forms. A secondary presentation following the primary presentation for the first cell of the matrix in Figure 2 is the example of contextual elaboration, which means presenting contextual or historical background in addition to the generality. Proceeding in this manner, Merrill enumerates several different secondary presentation forms pertaining to the content of the matrix cells.

The final step in finding a base for designing instruction is combining the performance taxonomy and the subject matter taxonomy with the presentation form taxonomy. Thus, provided for each specific objective in the performance-content matrix is the general design for instruction appropriate for achieving that objective.

Merrill's approach is based on the assumptions formulated by Gagné (1965) and later applied by Gagné and Briggs (1979). Each objective has its own conditions for learning the specified content and performance. If this design of instruction, which is prescribed on the basis of content type and performance level, is valid for all learning situations at school, it can be questioned whether a further development of instructional design is in fact necessary. In my opinion, however, there are reasons for further development, based upon several unanswered questions.

First, acquisition of knowledge and skills is intended to improve understanding of reality and to solve problems. The knowledge presented at school concerns this reality and the instructional designer has to decide how an aspect of that reality will be presented. There are several possibilities, including a part of the reality itself, or some form of transmediated representation: a picture, a movie, a diagram, a model, a formula or a simulation.

The question of which way reality will be best represented during instruction needs to be answered together with questions of whether different representations should be presented and in what sequence. Finally, the effect of the particular representation of reality on the mental representation and its retention should be studied.

A second reason for further development of instructional theory is the need for designing questions (or formulating problems) which trigger the learning of problem solving procedures, together with developing a body of declarative knowledge.

Finally, more research needs to consider the design of instruction for integrating knowledge and skills, which means integrating concepts and principles for solving problems. Answers to these questions can be given derived through systematic experiments concerned with both assessing knowledge representation and also providing insight into information processing.

Above all a learning theory is necessary. How can the transitions from novice to experts be described and how can expert behavior be predicted? How much practice is necessary and how can transfer be effected? 
In the learning process concepts play a crucial role. Concepts are supposed to be fundamental for thinking, because unlimited numbers of different things can be treated as similar for some purpose. Therefore, the teaching and acquisition of concepts is discussed first.

\section{The teaching and acquisition of concepts}

Having acquired a concept means being able to categorize objects, situations or states. Such categorizing is based on knowing a conceptual rule. Instructional design principles for teaching concepts have been developed primarily with reference to findings from experiments on learning well-defined concepts (e.g. Klausmeier and Allen, 1978; Merrill and Tennyson, 1977). Of central importance has been the content structure of the conceptual rule. However, in designing effective instruction, problems that deal with knowledge representation arise. For example, in which way should the object to be classified be actually represented? Moreover, the relevant or defining attributes should be shown such that the relationship between the object represented in instruction and the object in reality is clear.

Nearly all theories of concept learning make the assumption that representation of concepts in memory is based on attributes. Rosch (1977) has shown that for sensorially perceptible objects, the base category has three unique characteristics. First, members of the base category tend to look similar, e.g. birds. Second, the physical interactions with different members of the same basic-level category are similar, e.g. chairs. A third characteristic is that a basic-level category has many describable attributes. These characteristics are not valid for the members of a superordinate category. In contrast, it is difficult to find distinctive attributes for members of categories like furniture, games, etc. The attributes of members of the base-level categories are both relevant and irrelevant. Which objects or examples should be presented in instruction, assuming that instruction for concept learning starts with examples from base categories? The answer to this question comprises two "extreme" cases. Firstly, present a realistic example of a category, which has both relevant and irrelevant attributes and which should function as a prototypical example. Secondly, present a graphic example of a category, the irrelevant attributes of which are removed as far as possible - a generic example. Whether this can function as a prototype is not clear.

In the case of natural objects (such as plants and animals), irrelevant attributes are numerous, and presentations in instruction often greatly deviate from the object in reality. The instructional developer is thus confronted with a substantial design problem: what are the criteria for developing a best example? Several possibilities exist, e.g. the relevant attributes should be clear; irrelevant attributes which correlate strongly with the object ( .80 to 1.00 ) should be part of the best 
example. Including such irrelevant attributes in instruction helps develop the clearest case or prototype in memory.

The prototype is assumed to be a point in a fuzzy, multi-dimensional conceptual space. Its position is dependent on the values the object has on different dimensions. Neumann (1977) assumes that the prototype is situated at the intersection of dimension values most frequently occurring. The prototype doesn't exist. It is conceived as a prototype effect, being a consequence of the conceptual structure. During learning of well-defined concepts, irrelevant attributes are also stored since they accompany the relevant ones and thus become associated.

As an example, Compositae, a family of plants including the daisy, are defined by a florescence having either one or two types of florets. In fact, this represents an inclusive disjunctive concept. Categorising plants as members of this family requires careful inspection. However, because the florescence has a typical form, called a "head", categorising even by the layman based on this irrelevant attribute generally is correct. Should this irrelevant attribute not be mentioned during instruction, it nevertheless may be stored during the learning process. My suggestion is to isolate these typical attributes in instruction.

Merrill (1983) formulates the divergence rule which states that the form of the instances for a particular generality should be divergent. I suggest the additional point that irrelevant salient attributes should always be included in instruction, because storing these attributes may influence retention positively. But then a new problem arises. How many examples are necessary for realizing prototype effects? And how many examples are necessary for the formation of a valid respresentation of the category? Recent research by Dijkstra, Leemkuil and Ranzijn (1987) tries to answer these questions. They used "ecologically valid" school subject matter and studied the effect of the two variables mentioned on the ability to classify, i.e. procedural knowledge, and on the verbal or declarative knowledge. The concept windflower (flowers of which the pollen is transported by the wind) was used. One or four examples were presented. The dispersion of the examples could be either narrow or broad. In the first case, the irrelevant attributes of the examples were similar (cock's foot, stinging nettle, hazel and reed). In the second case the irrelevant attributes diverged (hazel, dock, leaved tree and grass).

The findings showed the number of examples is rather irrelevant for prototype abstraction. Only one example showing the defining and highly correlated variable attributes is sufficient. The results further showed the dispersion of examples improved the ability to classify. For the composition of the instructional design for teaching concepts, these findings mean that the instruction should start with the presentation of a best example, being constructed or selected by the teacher or groups of specialized teachers. The presentation of the best example should be followed by the presentation of examples, the variable attributes of which have different associated weights. This enhances the procedural skill of classifying. 
Tennyson, Youngers and Suebsonthi (1983) were the first instructional technologists to use prototype theory. They postulate a model for the acquisition of concepts that has two components: the formation in memory of information representative of a concept class (i.e. declarative knowledge) and also the development of a cognitive skill with which to use the representative information for generalization and discrimination (i.e. procedural knowledge).

In a more recent publication, Tennyson and Cocchiarella (1986) make a distinction between successive and coordinate concepts. In the latter case learning includes the development of skills to generalize within a concept class and to discriminate between concepts. Thus, coordinate concepts comprise exclusive disjoint categories. Sometimes knowledge of a principle makes categorization of objects in disjoint categories possible. This leads to the following question: what is the design of instruction in this case and is prototype formation possible?

For an adequate answer some problems have to be solved. Take an example from biology. Plants may be categorized on the principle of pollination. Different ways for the transport of male cells are known, such as by insects, by wind, by birds, or, in more rare cases, by water or by bats. Based on about six attributes, an expert biologist decides into which category a plant belongs, based on type of pollination. Because millions of plants belonging to different families comprise these categories, the instructional designer needs to consider which examples would be best used in instruction. Unfortunately, there may be insufficient overlap even in relevant attributes so prototype effects will be impossible. As Tennyson and Cocchiarella state, conceptual knowledge implies complete formation of attributes, i.e. a prototype. However, because there is so much divergence among the irrelevant attributes in this example and moreover categorization is based on a principle, I propose a somewhat revised model of concept learning including, first, the formation of a schema representing the principle, second, the formation in memory of the information representative of a concept class (either as a single case or as a prototype), and third, the development of the cognitive skill to use this representative information for generalization and discrimination.

For designing instruction this means presenting a diagram representation of the principle, showing only the relevant attributes, together with a real life illustration of the process. This can be accomplished by movie or interactive video. In this way, the relevant attributes of the objects are depicted and can be compared for different categories. At the end of instruction new categorization items are presented until the criterion is reached.

The examples of learning to categorize describe two processes. The first process is learning to categorize "similar" objects in one category. The second process is leaming to partition a universe of discourse in disjoint categories. The partition is based on general principles, often describing a process chronologically: life cycle, growth, pollination, florescence, etc. Instruction for learning to categorize is realized by first presenting an example, being a member of one of 
Table 1. Partial production system for answering the questions "Is $x$ a $P$ or $Q$ or R?"

IF the goal is to answer whether $\mathrm{x}$ is a $\mathrm{P}$ or $\mathrm{Q}$ or $\mathrm{R}$,

$A N D \cap Q \cap R=\varnothing$

$A N D x \in(P \cup Q \cup R)$

THEN formulate as subgoals to answer whether

$x$ is a $P$

$x$ is $a$

$x$ is a $R$

the disjoint categories. The relevant (defining) attributes are made discriminable and the principle is explained. The verbal (declarative) knowledge and the procedure for classification (procedural knowledge) are exposed. If the concept is inclusive disjunctive, then the instructor decides to present more examples. The second instructional step is to present an example of another disjoint category from the same universe of discourse, based on a distinct appearance of the principle. Again the relevant attributes are made discriminable and the principle is explained. From that moment generalization to new categories is possible. A typical instruction reads: there are more categories based on other "appearances of the principle". Such instructions refer to conditional knowledge. This means in this case that teachers and students have a notion that different processes can be governed by the same principle, and a notion that the outcome of a process can be categorized differently. Conditional knowledge is necessary for efficient storing of both declarative and procedural knowledge.

Psychologically, a concept exists when two or more objects or situations share common associates in memory (Richards and Goldfarb, 1986). Improvement in performance on concept learning tasks is attributable to the establishment of a constellation of relevant and irrelevant attributes in the form of either a prototype or a schema, along with the development of an executive system. Within this system, questions of the following form are answered: "Is this object an exemplar of the category 0 ?" The complete production system is described by Richards and Goldfarb for single concepts. For coordinate relationships, a production needs to be added, which is shown in Table 1. The system becomes proceduralized (Anderson, 1982) after sufficient classifications. The speed of application of the productions is part of the achievement criterion.

\section{Integrating procedures, principles and concepts}

Concepts are based on relations between objects or attributes of objects. The label class concept is used in case of similarity of objects. In other cases concepts are labelled relational. A relational concept specifies the relations between elements 
of different sets or of the same set. Classification can be based both on relations and principles. For example, categorising a subset of men as fathers is done on the basis of the relation " $a$ is the father of b"; categorizing flowers as windflowers is done based on the principle: "If the stamen has a specific form, then pollination is caused by wind". Often concepts are scalar, which means based on a partition of a variable. For example, labelling people as rich or poor does not result in welldefined categories.

It is impossible to understand reality without the integration of class and relational concepts, together with principles. Understanding is possible by using knowledge in solving problems and training in problem solving procedures. These problems should be formulated in such a way that a student is able to discover basic conceptual patterns, which have been presented in instruction. A basic conceptual pattern is conceived as a representation of an object - either as a prototype or a generic object - and its conceptual rule; a representation of different objects and the relation between these objects, or a (schematic) representation of a principle. In order to be able to discover the basic conceptual patterns the problem situation often has to be restructured. Then the student can decide in which category the problem belongs (Wertheimer, 1945). Once a problem is recognized as belonging to a certain category, a solution procedure can be applied. The instructional designer has to show the basic conceptual patterns necessary for the solution of a problem belonging to a certain category and has to decide which variations in problems of the same category are necessary for the formation and retention of declarative knowledge in memory. To illustrate this, consider the following series

$$
\begin{array}{llllll}
2 & 5 & 8 & 11 & 14 & \ldots
\end{array}
$$

The question, "Which element comes next?" is easily answered by determining the regularity of the series. This is done by calculating the difference between the successive elements. This procedure is adequate for solving the problem. Knowledge of the set of positive integers and the operations of adding and subtracting (procedural knowledge) seems sufficient.

Changing the problem to "Which is the sixtieth number?" makes the previous procedure for solving the problem inadequate. A more efficient procedure is necessarily based on a relational concept. Consider the following series

$\begin{array}{rrrrrrr}\mathrm{x} & 1 & 2 & 3 & 4 & 5 & 6 \\ \mathrm{y} & 2 & 5 & 8 & 11 & 14 & \ldots\end{array}$

If the elements of the first series and the elements of the second series are considered as the objects of ordered pairs in the sequence given, then it can easily be seen that they belong to a function, because no two pairs have the same first element. A simpler description than (2) is found by calculating the first row of differences and concluding that these differences are equal.

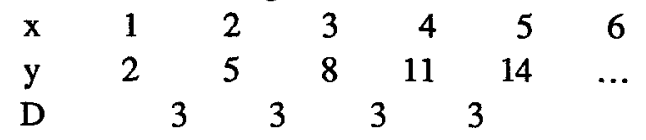


This means the function is linear and has the form

$$
y=a x+b
$$

The real formula of this function is found by using the procedure of solving an equation with two unknown elements

$$
\begin{aligned}
& \text { for } x=1: 2=a+b \\
& \text { for } x=2: \frac{5=2 a+b}{-3=-a} \\
& : a=3 \text { Substition of a yields } \\
& b=-1
\end{aligned}
$$

and the description of the function is

$$
\mathrm{y}=3 \mathrm{x}-1
$$

Using this description, each element can be found. And so the sixtieth element is $3 * 60-1=179$.

Now consider the following problem in physics. The coordinates of an object 0 , which is moving uniformly rectilinearly, are depicted at seconds $1,2,3,4$ and 5 as

$$
\begin{array}{llllll}
2 & 5 & 8 & 11 & 14 & \ldots
\end{array}
$$

Which is the coordinate on the sixtieth moment? Because the speed of an object, moving uniformly rectilinearly is the (constant) ratio of displacement and time period, this problem can be solved as follows:

Let $x_{t}$ denote the coordinate at moment $t$

$$
\mathrm{v}=\frac{\Delta \mathrm{x}}{\Delta \mathrm{t}}=\mathrm{constant} \quad \Delta \mathrm{x}=\mathrm{v}^{*} \Delta \mathrm{t}
$$

It follows

$$
\begin{aligned}
& \text { And }\left(x_{1}-x_{1}\right)=v(t-1) x_{1}=x_{1}+v *(t-1) \\
& \qquad \begin{aligned}
x_{60} & =2+3(59) \\
& =179
\end{aligned}
\end{aligned}
$$

Because $x_{1}$ and $(t-1)$ are constants it can be seen that (8) has the same form as (4). The answer to the actual problems in mathematics and physics are indeed the same.

What is happening psychologically is the integration of declarative knowledge, both concerning concepts and principles. The first is the integration of the first number series with the relational concept function. The series becomes the range of the function (Rf). The second is the integration of a physics principle and the relational concept function: the speed of a uniformly rectilinear moving object is constant. This can be understood by solving problems like those mentioned above, but the formulation of the problem is essential for the integration of the principle and the concept. Presenting the following pattern $n(g)$ in the problem formulation makes recognition of a linear function quite easy and facilitates integration. Asking the position coordinate of a uniformly rectilinear moving object 
with a speed of $3 \mathrm{~m}$ per second at the sixtieth moment, when its position is 2 at the first second, makes recognition quite difficult.

$\begin{array}{rrrrrrr}\mathrm{t} & 1 & 2 & 3 & 4 & 5 & 6 \\ \mathrm{x}_{\mathrm{t}} & 2 & 5 & 8 & 11 & 14 & \ldots\end{array}$

The problem requires further categorization of an abstract object. The object becomes a generic object. Its attributes are removed, except that the object can be displaced and has a coordinate at a certain moment. Thus, it is integrated in the principle and in the relational concept simply as a point.

\section{Summary}

The problems of instructional design firstly are concerned with (1) the representation of reality in instruction and its effect on both the nature of mental representation and on long term retention, and (2) the search for basic patterns of concepts and principles, which are presented in instruction and made recognizable in the formulation of problems. This is critical for making the necessary integration. Procedures as production systems are well described. However, the representation of declarative knowledge and integration of existing knowledge with new knowledge as a result of instruction needs more investigation.

\section{Acknowledgements}

This paper was presented in the symposium "International Perspectives on Meta-Learning Theories and Instructional Design", Frank Farley (Chair), Annual Meeting of the American Educational Research Association, New Orleans, April 1988.

\section{References}

Anderson, J. (1982). Acquisition of cognitive skill. Psychological Review, 89(4), 369-406.

Boume, L. E. (1970). Knowing and using concepts. Psychological Review, 77, 546-556.

Bruner, J. S., Goodnow, J. J. and Austin, G. A. (1956). A study of thinking. New York: Wiley.

Dijkstra, S., Leemkuil, H. and Ranzijn, F. J. A. (1987). Instruction for leaming natural concepts. Paper presented at the meeting of the European Association for Research on Learning and Instruction, Tübingen, West-Germany.

Gagné, R. M. (1965). The conditions of learning (1st ed.). New York: Holt, Rinehart and Winston. Gagné, R. M. (1985). The conditions of learning (4th ed.). New York: Holt, Rinehan and Winston.

Gagne, R. M. and Briggs, L. J. (1979). Principles of instructional design. New York: Holt, Rinehart and Winston.

Klausmeier, H. J. and Allen, P. S. (1978). Cognitive development of children and youth: a longitudinal study. New York: Academic Press.

Lakoff, G. (1987). Cognitive models and prototype theory. In U. Neisser (Ed.), Concepts and conceptual development. Cambridge: University Press.

Merrill, M. D. (1983). Component display theory. In C. M. Reigeluth (Ed.), Instructional design theories and models. Hillsdale, N. J.: Lawrence Erlbaum Associates. 
Merrill, M. D. (1987). The new component design theory: instructional design for courseware authoring. Instructional Science, 16, 19-34.

Merrill, M. D. and Tennyson, R. D. (1977). Concepts teaching: an instructional design guide. Englewood Cliffs, N. J.: Educational Technology.

Neumann, P. G. (1977). Visual prototype formation with discontinous representation of dimensions of variability. Memory and Cognition, 5, 187-197.

Reigeluth, C. M. and Stein, F. (1983). The elaboration theory of instruction. In C. M. Reigeluth (Ed.), Instructional design theories and models. Hillsdale, N. J.: Lawrence Erlbaum Associates.

Richards, D. D. and Goldfarb, J. (1986). The episodic memory model of conceptual development: an integrative viewpoint. Cognitive Development, 1, 183-219.

Rosch, E. (1977). Classification of real-world objects: origins and representations in cognition. In P. N. Johnson-Laird and P. C. Wason, Thinking. Cambridge: University Press.

Rosch, E. (1978). Principles of categorization. In E. Rosch and B. B. Lloyd (Eds.), Cognition and categorization. Hillsdale, N. J.: Lawrence Erlbaum Associates.

Tennyson, R. D., Youngers, J. and , Suebsonthi, P. (1983). Concept learning by children using instructional presentation forms for prototype formation and classification skill development. Journal of Educational Psychology, 75(2), 280-291.

Tennyson, R. D. and Cocchiarella, M. J. (1986). An empirically based instructional design theory for teaching concepts. Review of Educational Research, 56(1), 40-71.

Wertheimer, M. (1945). Productive thinking. New York: Harper and Brothers. 